\title{
Michael Heinrich
}

\section{Kosovo 1999}

54 Jahre nach dem Ende des Zweiten Weltkriegs befindet sich Deutschland wieder im Krieg; und dieser Krieg wird nicht von einer national-konservativen, sondern von einer rot-grünen Regierung geführt, die die konservative Vorherrschaft im September 1998 durch einen fast erdrutschartigen Wahlsieg beendete. Auch in anderen NATO-Ländern wie England, Frankreich, Italien oder den Niederlanden, sind es sogenannte Mitte-Links-Regierungen, die ihre $\mathrm{Zu}$ stimmung zum Krieg gegeben haben. Was (West-)Deutschland angeht, so gehörte in den 80er Jahren ein großer Teil der rot-grünen Basis zur Friedensbewegung, die gegen die Stationierung neuer Raketen opponierte. Jetzt folgt eine Mehrheit der bündnisgrünen Parteimitglieder dem rot-grünen Regierungskurs, wie auf dem Sonderparteitag der Grünen im Mai in Bielefeld deutlich wurde. $\mathrm{Da} ß$ Bomben aus humanitären Gründen geworfen werden, daß angeblich gar nichts anderes übrig bleibt, um die Rechte der Kosovo-Albaner zu schützen, scheint Teile der ehemaligen (West-) Friedensbewegung zu überzeugen - im Osten sind die Vorbehalte gegen den Bombenkrieg größer. Die Überzeugung von der moralischen Überlegenheit und politischen Richtigkeit des eigenen Tuns ist so groß, da $\beta$ den Kritikern der Bombardierungen häufig unterstellt wird, sie wollten überhaupt nichts gegen ethnische Vertreibung unternehmen oder stünden auf der Seite der "mörderischen Serben« und des »Monsters Milosevic«. Entweder am Elend von Hunderttausenden mitschuldig zu werden oder Bomben auf Belgrad werfen, das sei die Alternative. Dies zumindest suggeriert die große Mehrheit der zwar nicht gleichgeschalteten aber trotzdem weitgehend gleich berichtenden Medien. Und wenn sich dann doch einmal ein Artikel, der den NATO-Krieg grundsätzlich in Frage stellt, auf die »Meinungsseite « eines ansonsten durchaus kritischen und liberalen Blattes wie der Berliner Zeitung verirrt, dann wird sogar der nächtliche Druck gestoppt und der allzu kritische Artikel durch einen Beitrag über die Fusionspläne der Telekom ersetzt - »aus Aktualitätsgründen $\ll$.

I.

Auch wer es für legitim erachtet, Serbien zu bombardieren, um die Situation der Kosovo-Albaner zu verbessern, kann nicht übersehen, daß gerade dieses Ziel massiv verfehlt wurde. Den KosovoAlbanern, die seit der Aufhebung des Autonomiestatuts vor zehn Jahren von der serbischen Zentralregierung zunehmend unterdrückt werden, geht es seit Beginn des Bombardements schlechter 
denn je. Die Vertreibungen großen Stils setzten mit den Bombardements ein; sie zu verhindern, ist der NATO Kriegsmaschinerie gerade nicht gelungen. Im Gegenteil, sie wurden als Waffe benutzt, da gegen die Luftüberlegenheit der NATO-Jets nichts auszurichten war. Das ist zwar zynisch und menschenverachtend; es ist aber ein Kriegsmittel, das die Strategen in Brüssel, Washington oder auf der Hardthöhe in Bonn hätten einkalkulieren müssen. Es kann freilich sein, daß sie dies taten, und die Folgen kaltblütig in Kauf genommen haben.

Daß der militärische Erfolg der NATOAngriffe die hochgesteckten Anfangserwartungen bisher nicht erfüllte, ist nicht überraschend, wenn man berücksichtigt, daß die jugoslawische Verteidigungsstrategie seit 50 Jahren darauf ausgerichtet ist, den Angriff eines militärisch und technologisch überlegenen Gegners (wobei in der Vergangenheit in erster Linie die Sowjetunion als Aggressor vermutet wurde) möglichst ins Leere laufen $\mathrm{zu}$ lassen, indem sowohl Kommando- als auch Versorgungsstrukturen der Armee weitgehend dezentralisiert wurden, so daß der Krieg nur durch verlustreiche Kämpfe auf dem Boden zu gewinnen ist.

Der geringe militärische Erfolg ließ die NATO dazu übergehen, auch zivile Ziele zu zerstören, von Donaubrücken (im Norden des Landes, weit weg vom Kosovo) über Zigarettenfabriken und Heizkraftwerke bis zu Fernsehsendern und Wasserwerken. Damit wird aber nicht nur die Infrastruktur sowohl Serbiens wie des Kosovo zerstört, mit der Bombardierung von Chemiefabriken und Raffinerien sowie der Verwendung uranhaltiger Geschosse sind langanhaltende Umweltschäden in der gesamten Region, radioaktiv verseuchte Gebiete und gesundheitliche Risiken für die Bevöl- kerung programmiert - eine humanitäre Katastrophe als direkte Folge der NATO-Bombenpolitik. Durch den Einsatz von Bodentruppen könnte die NATO den Krieg wohl militärisch gewinnen, aber nur um den Preis einer großen Anzahl von Opfern nicht nur unter den eigenen und den gegnerischen Soldaten, sondern vor allem unter der Zivilbevölkerung.

Je offensichtlicher es wird, daß dieser Krieg den Menschen, denen er angeblich helfen sollte, nichts nutzt, sondern im Gegenteil ganze Gesellschaften traumatisiert, desto stärker verschieben sich die angebotenen Rechtfertigungen. Es könne doch nicht angehen, daß Milosevic triumphiert, daß ein Herrscher, der die Menschenrechte derart grob verletzt, ungeschoren davon kommen könne. Henry Kissinger hat dieser Logik Ausdruck verliehen: Die NATO-Strategie sei politisch verrückt, müsse aber nun bis zum Sieg fortgesetzt werden, um nicht das Gesicht zu verlieren. Ein Sieg soll diesem Krieg zur Legitimation verhelfen. Dabei geht es längst nicht mehr um Hilfe für die Kosovo-Albaner, sondern um ein Strafgericht. Die NATO-Jets sind in dieser Inszenierung der Clintons, Fischers, Blairs und Schröders die apokalyptischen Reiter, die gen Serbien ausgesendet werden.

\section{II.}

Daß die NATO diesen Krieg aus rein humanitären Gründen führe, ist schon deshalb wenig plausibel, weil die Diskriminierung der Kosovo-Albaner, die seit 1989 kontinuierlich zugenommen hat, bis 1998 von den NATO-Staaten eher heruntergespielt wurde - wollte man doch nicht allzu viele politische Flüchtlinge aufnehmen. Und es spricht nicht gerade für den hohen Stellenwert der vorgegebenen humanitären Ziele, 
daß nicht die geringsten Ansätze eines Umdenkens $\mathrm{zu}$ bemerken sind, wenn offensichtlich wird, daß sie mit den angewandten Methoden nicht erreicht werden können.

Auch das andere Rechtfertigungsargument, daß man den Menschenrechten auch durch äußere Intervention Geltung verschaffen müsse, daß man Staaten und deren Regierungen für das Unrecht, das sie ihrer eigenen Bevölkerung zufügen, zur Rechenschaft ziehen sollte, ist nicht besonders schlüssig: Zur Durchsetzung von Rechtsverhältnissen gehört nämlich nicht nur, daß der Rechtsbrecher verfolgt und bestraft wird, sondern daß dies auch innerhalb eines rechtlichen Rahmens geschieht. Die bescheidenen Ansätze eines solchen Rahmens, die sich innerhalb der UNO finden, wurden aber von der NATO ganz bewußt umgangen. Stattdessen präsentiert sich die NATO (nur zur Erinnerung: es handelt sich dabei um ein Verteidigungsbündnis, das seine Mitgliedsstaaten gegen äußere Angriffe schützen soll) in einer Person als Ankläger, Richter und Urteilsvollstrecker. Der Cowboy, der den bösen Schurken endlich umlegt, mag zwar eine sympathische Figur aus diversen WesternFilmen sein, mit der Durchsetzung von Rechtsverhältnissen im internationalen System am Ende des 20. Jahrhunderts hat ein solches Vorgehen allerdings nichts zu tun.

Daß dieser Cowboy namens NATO nur ein (gerechtfertigter) Vorgriff auf eine Weltbürgergesellschaft sei, in der auch Regierungen von der internationalen Gemeinschaft für Menschenrechtsverletzungen an ihren Bürgern zur Rechenschaft gezogen werden, wie Jürgen Habermas in seinem ZEIT-Essay meinte (Bestialität und Humanität, DIE ZEIT Nr. 18, 29.5.1999), unterstellt in stu- pender Naivität erstens, daß eine solche »Weltbürgergesellschaft« überhaupt angestrebt wird. Eine solche Unterstellung wird schon durch die langjährige Praxis der Krieg führenden Länder dementiert. In Lateinamerika und Asien wurden (und werden) von den USA brutale Diktaturen genauso unterstützt wie Regierungen, die Bürgerkriege gegen Teile des eigenen Volkes führen. Und das NATO-Mitglied Türkei, das am Angriff auf Serbien beteiligt ist, hält nicht nur halb Zypern besetzt und betreibt dort eine Politik der ethnischen Spaltung, es verweigert im eigenen Land den Kurden jede Autonomie. Die türkische Armee brennt seit mehr als einem Jahrzehnt ganze kurdische Dörfer nieder, vertreibt die Menschen aus der Region und foltert vermeintliche und tatsächliche Unterstützer der kurdischen Autonomiebestrebungen. Wer Mitglied der NATO ist, kann im eigenen Land offenbar ungestraft morden und vertreiben.

Von Habermas und vielen anderen wird dieser Einwand beiseite geschoben, indem einfach erklärt wird, daß man doch nicht an einer Stelle auf Hilfe verzichten könne, nur weil sie an anderer Stelle nicht möglich sei. Wieso es aber soviel schwieriger sein soll, Druck auf den NATO-Partner Türkei auszuüben, als Bomben auf Serbien zu werfen, dafür fehlt bislang jede Erklärung. Anstatt zumindest politischen Druck auszuüben, erhält die Türkei von NATO-Mitgliedern wie den USA und Deutschland großzügige Militär- und Finanzhilfe, die nicht zuletzt für den »schmutzigen Krieg « im eigenen Land benötigt wird. Humanität und Recht sind hier ganz offensichtlich anderen Interessen untergeordnet, so daß die Vermutung legitim ist, daß es auch im Fall Jugoslawien noch um mehr und anderes geht. 
III.

Daß die öffentlich geäußerten Gründe für diesen Krieg auf schwachen Füßen stehen, scheint auch den Krieg führenden Politikern klar zu sein. Daher ist es kein Zufall, daß der Gegner zum absolut Bösen aufgebaut wird, gegen den jedes Mittel recht ist. Wie schon Saddam Hussein wird jetzt auch Milosevic zum neuen Hitler erklärt. Beide Diktatoren sind vom Westen, solange sie als regionale »Stabilitätsfaktoren « in der »neuen Weltordnung « galten, unterstützt worden; die repressive Politik wurde verharmlost. Das »Böse« hat man erst entdeckt, als man sie loswerden wollte. Im Fall Jugoslawien geht die Dämonisierung aber weit über Milosevic hinaus. Nicht nur in der Boulevardpresse erscheinen »die Serben« schon fast als ein einzig Volk von Mördern und Vergewaltigern, die ein neues »Auschwitz« hervorbringen. Daß die serbische Opposition gegen Milosevic in den letzten Jahren erheblich größer war, als sie es in Deutschland jemals gegen Hitler war, ist da nicht weiter wichtig. Nicht nur das Böse ist jetzt identifiziert und rechtfertigt jeden Kriegseinsatz; gerade für die deutschen Politiker kommt mit solchen Konstruktionen etwas Wichtiges hinzu: der Faschismus wird ein zweites Mal geschlagen und endlich stehen die Deutschen auf der richtigen Seite.

Daß die Unterdrückung der KosovoAlbaner, ihre jetzt stattfindende Vertreibung, die Morde und Vergewaltigungen schlimmste Verbrechen sind, wird keineswegs verharmlost, wenn man gegen die Hitler- und Auschwitz Vergleiche der offiziellen Kriegspropaganda die Singularität der Verbrechen des Nationalsozialismus in Erinnerung ruft: die Nazis haben (mit Zustimmung und Unterstützung der Mehrheit der »ganz normalen Deutschen «) Europa mit Krieg überzogen sowie einen bürokratisch geplanten und industriell ausgeführten Massenmord an Millionen von Juden und Zigtausenden von Roma und Sinti, Kommunisten, Schwulen und »lebensunwertem Leben« begangen.

Auch wenn nun allenthalben so getan wird: Milosevic ist nicht der urplötzlich aufgetauchte Schurke, der allein für alles Übel verantwortlich ist. Jugoslawien war auch schon vor 1989 ein fragiles Gebilde. Weniger wegen der vielen Nationalitäten, sondern vor allem wegen der enormen wirtschaftlichen und kulturellen Unterschiede, die es innerhalb dieses Staates gab: während sich Slowenien Hoffnung machen durfte, wirtschaftlich irgendwann einmal an den EU-Durchschnitt heran zu kommen, war der Süden Jugoslawiens eher mit einem Land der »Dritten Welt« vergleichbar. Bis in die Mitte der 80er Jahre ermöglichte nicht nur ein kompliziertes staatliches Institutionensystem einen gewissen Ausgleich der divergierenden Interessen, wichtig für den Zusammenhalt war auch der äußere Druck in Gestalt des Ost-West Gegensatzes, der es für alle Beteiligten deutlich machte, daß die internen Konflikte nicht eskalieren durften, sollte die relativ unabhängige und komfortable Mittelposition zwischen Ost und West - Neutralität, führendes Mitglied in der BlockfreienBewegung, Politik der »self-reliance « nicht verloren gehen. Mit der Umwälzung der weltpolitischen Lage und den zunehmenden wirtschaftlichen Schwierigkeiten - die durch die Auflagen der Institutionen von Bretton Woods vergrößert wurden - veränderte sich die Situation aber grundlegend. Für die entwickelteren wirtschaftlichen Regionen erschien jetzt ein Ausscheiden aus dem gesamtjugoslawischen Staatsverband 
sowohl politisch möglich als auch ökonomisch vorteilhaft zu sein. In diesem Prozeß wurden dann von allen Seiten die verschiedenen Nationalismen mobilisiert, die nicht nur brauchbare Feindbilder, sondern auch die Legitimation für die alleinige Nutzung vorhandener Ressourcen liefern.

Die zentrifugalen Kräfte wurden Anfang der 90er Jahre gerade vom Westen - und hier insbesondere vom neu vereinigten Deutschland - kräftig gefördert. Mit der frühzeitigen staatlichen Anerkennung von Kroatien und Slowenien, die in der EU 1991 vom deutschen Außenminister Genscher durchgesetzt wurde, brach die komplizierte Konstruktion Jugoslawiens schnell zusammen und die Konflikte, die heute durch den Einsatz von Bomben gelöst werden sollen, wurden kräftig angefacht: jede Minderheit, die sich von der $»$ repressiven « Mehrheit lossagte und auf Selbstbestimmung pochte, verweigerte ihren eigenen Minderheiten genau diese Selbstbestimmung. Auf diese Gefahr hatte der damalige UNO-Generalsekretär Perez de Cuellar in einem warnenden Brief an Genscher hingewiesen erfolglos. Kroatien befreite sich von der serbischen Vorherrschaft und diskriminierte gleichzeitig die serbische Minderheit im eigenen Land. Serbien geiBelt zwar die Unterdrückung der serbischen Minderheiten andernorts, geht aber genauso rücksichtslos gegen die ungarische und die albanische Minderheit im eigenen Land vor. Der Versuch, das zu erhalten, was es in Jugoslawien vor 1991 an Koexistenz der verschiedenen Ethnien und Religionen und der verschieden entwickelten Landesteile gab, wäre ein aufgeklärtes, demokratisches und europäisches politisches Projekt gewesen. Man hat es mit schmählicher Beteiligung der deutschen $\mathrm{Au}$ -
Benpolitik sabotiert - und es dann isoliert in Bosnien-Herzegowina durchsetzen wollen. Dort, in einem »Staatswesen «, das noch niemals existierte, umgeben von einem kroatischen und einem serbischen Staat, hat es nicht funktionieren können.

Daß der Westen Anfang der 90er Jahre bei der Auflösung Jugoslawiens kräftig mitmischte, hatte vor allem mit geopolitischen Interessen $\mathrm{zu}$ tun: die weitere Entwicklung der Sowjetunion bzw. ihrer Nachfolgestaaten war unklar und die Entstehung eines neuen antiwestlichen Blockes sollte auf jeden Fall verhindert werden. Dieses Interesse spielt bis heute eine wichtige Rolle bei der NATOPolitik: zumindest außerhalb der GUSStaaten wird von der NATO die Regelungskompetenz beansprucht. Das macht die neue NATO-Doktrin deutlich, die Interventionen außerhalb des ursprünglichen Bündnisgebietes und ohne Zustimmung der UNO vorsieht. Vor allem bei den europäischen NATOStaaten dürfte noch ein weiteres, ganz eigenes Motiv von erheblicher Bedeutung sein: die Angst vor Flüchtlingsströmen, wie sie schon der Bürgerkrieg in Bosnien hervorgebracht hat.

Wer der NATO eine vorwiegend moralische Motivation unterstellt, wendet hier ein, daß diese Interessen wohl kaum einen derart kostspieligen und langwierigen Krieg erklären könnten (so auch Habermas in seinem oben erwähnten Artikel). Nun sieht es tatsächlich so aus, als ob die NATO diesen Krieg keineswegs langfristig geplant hätte. Daß die NATO-Strategen offenbar nur mit einem kurzen Krieg gerechnet haben, wird schon an der mangelnden medialen Kriegsvorbereitung deutlich: im Unterschied zum Golfkrieg von 1991 setzte die entsprechende Propagandaschlacht erst mit der militärischen 
Auseinandersetzung ein und nicht schon Wochen vorher. In Rambouillet wurde allerdings auch deutlich, daß von Jugoslawien eine bedingungslose Kapitulation erwartet wurde, einschließlich der Zustimmung zur Stationierung einer defacto Besatzungstruppe nicht nur im Kosovo sondern im gesamten jugoslawischen Staatsgebiet, wie aus dem inzwischen auch hierzulande in der Presse bekannt gewordenen Annex B zum Vertragsentwurf hervorgeht. Die NATO glaubte offensichtlich, alle Bedingungen diktieren zu können, sofern sie nur glaubwürdig mit der Bombardierung droht und diese eventuell auch ein paar Tage durchführt. Nachdem sie den Krieg aber nun einmal begonnen hat, muß ihn die NATO auch weiterführen, will sie, wie die beteiligten Regierungen selbst betonen, ihre »Glaubwürdigkeit« - und das heißt: ihr zukünftiges Drohpotential gegenüber aufmüpfigen Potentaten oder Regierungen, die sich weigern, nach der NATO-Pfeife zu tanzen - nicht beschädigen. Daß sich dieser Krieg als länger und kostspieliger erweist, als ursprünglich erwartet wur$\mathrm{de}$, ist kaum eine überzeugende Begründung dafür, daß er nicht mit Interessen zu tun hätte und nur aus rein moralischen Gründen geführt werde.

IV.

Mehrere Auswirkungen dieses Krieges zeichnen sich bereits ab. Rußland, das bisher immer noch als Supermacht behandelt wurde, obwohl es längst keine mehr ist, wurde jetzt unübersehbar zur Regionalmacht zurückgestuft, der allenfalls ein kleiner, regionaler Herrschaftsbereich zugebilligt wird. Außerhalb dieses Bereichs erklärt sich die NATO als allein zuständig, um das, was sie als Ordnung definiert, notfalls auch mit militärischer Gewalt durchzusetzen. Allein die NATO entscheidet (wie in der gerade beschlossenen neuen NATO-Doktrin unmißverständlich klargemacht wurde), ob sie bei diesem Prozeß die UNO (oder auch Rußland) mit einbezieht oder nicht. Die Ansätze einer »Partnerschaft für den Frieden « mit den mittel- und osteuropäischen Staaten, die die NATO nach 1991 zunächst verfolgt hat, können getrost vergessen werden. Die NATO setzt jetzt auf Interessen- und Machtpolitik, ohne einen Ausgleich zu suchen. Die einen werden in die NATO als volle Mitglieder aufgenommen, die anderen bleiben draußen. Dies gilt insbesondere für Rußland.

Die absehbare Niederlage Jugoslawiens ermöglicht der NATO, die gesamte Südflanke Rußlands von Westeuropa über die Balkan-Staaten und die Türkei bis hin zum Kaukasus und zum Kaspischen Meer zu kontrollieren. Das rohstoffreiche Zentralasien gerät ins Visier. In dieses geo-strategische Bild paßt auch, daß die NATO-Länder USA und Großbritannien nicht nur Jugoslawien sondern ebenso den Irak fast tagtäglich bombardieren. Auch dort mußten schon Zigtausende die »Verteidigung der Menschenrechte« durch Bombardements und Embargopolitik mit ihrem Leben bezahlen.

Für Deutschland bedeutet die Teilnahme an diesem Krieg einen in seiner Tragweite kaum zu überschätzenden Einschnitt. Es wird mit einem grundlegenden (im Grundgesetz auch verfassungsrechtlich verankerten) Tabu gebrochen, an das sich alle politischen Kräfte seit dem Ende des Zweiten Weltkrieges gehalten haben; ein Tabu, das noch 1991 eine militärische Beteiligung am Krieg gegen den Irak nicht einmal zum Diskussionspunkt werden ließ: Deutschland darf sich an keinem Angriffskrieg beteiligen. Noch entschei- 
dender als dieser Tabubruch ist der Sachverhalt, daß er unter einer rotgrünen Regierungskonstellation erfolgt. Zumindest Teile von SPD und Grünen sind die politischen Erben der Proteste gegen die Wiederbewaffnung in den fünfziger Jahren, gegen die Notstandsgesetze und den Vietnam-Krieg in den 60er Jahren und gegen die Nachrüstung in den 80er Jahren. Da der Krieg aber gerade von einer rot-grünen Regierung geführt wird, sind die politischen und gesellschaftlichen Räume, in denen sich eine Opposition zu diesem Krieg artikulieren kann, erheblich verkleinert. Damit ist es jetzt erstmals möglich gewor- den, über die Parteigrenzen hinweg (die PDS spielt in diesem Zusammenhang keine Rolle oder kann sogar noch als Alibi der möglichen Pluralität dienen) ein $»$ nationales Interesse « $\mathrm{zu}$ definieren, das auch unter Einsatz militärischer Mittel verfolgt wird. Rotgrün hat die »unverkrampfte Normalität«, die sich der scheidende Bundespräsident Roman Herzog wünschte, durchgesetzt: Unbelastet von der faschistischen Vergangenheit ist Deutschland jetzt wieder eine ganz normale Großmacht, mit der in Zukunft auch militärisch zu rechnen sein wird - an der Seite der NATOPartner, gegen den Rest der Welt. 